\title{
Pro-metastatic intracellular signaling of the elaidic trans fatty acid
}

\author{
KIYOMU FUJII ${ }^{1}$, YI LUO ${ }^{1,2}$, RINA FUJIWARA-TANI ${ }^{1}$, SHINGO KISHI $^{1}$, SONG HE$^{2}$, \\ SHUYUN YANG ${ }^{2}$, TAKAMITSU SASAKI ${ }^{3}$, HITOSHI OHMORI ${ }^{1}$ and HIROKI KUNIYASU ${ }^{1}$ \\ ${ }^{1}$ Department of Molecular Pathology, Nara Medical University, Kashihara 634-8521, Japan; \\ ${ }^{2}$ Department of Pathology, Nantong University Cancer Hospital, Nantong, Jiangsu 226361, P.R. China; \\ ${ }^{3}$ Department of Gastroenterological Surgery, Fukuoka University School of Medicine, Jonan-ku, Fukuoka 814-0180, Japan
}

Received October 17, 2016; Accepted December 2, 2016

DOI: $10.3892 /$ ijo.2016.3797

\begin{abstract}
Trans fatty acids (TFAs) are risk factors of cardiovascular disorders, and a few studies have reported the cancer-promoting effects of TFAs. In the present study, we examined the effects and signaling of elaidic acid (EA), a TFA, in colorectal cancer (CRC) cells. Oral intake of EA increased the metastasis of CT26 mouse CRC cells by inducing the expression of stemness markers nucleostemin (NS) and CD133. Mechanisms underlying EA-induced signaling were confirmed by determining the binding of EA to G-protein coupled receptor 40 (GPR40) and GPR120 by performing surface protein internalization assay. We found that c-SRC mediated EGFR transactivation was induced by the binding of EA to GPR40 and GPR120. Moreover, EGFR signaling upregulated NS and Snail expression and downregulated E-cadherin expression in wild-type APC-containing CT26 cells, and upregulated NS, Wnt5a and CD44 expression in APC-null HT29 cells. These results indicate that EA enhances the stemness and epithelial-mesenchymal transition of CRC cells. These results also indicate the prominent metastatic potential of EA-treated cancer cells and highlight the important implications of EA on public health.
\end{abstract}

Correspondence to: Professor Hiroki Kuniyasu, Department of Molecular Pathology, Nara Medical University, 840 Shijo-cho, Kashihara 634-8521, Japan

E-mail: cooninh@zb4.so-net.ne.jp

Abbreviations: CRC, colorectal cancer; TFA, Trans fatty acid; LCFA, long chain fatty acid; EA, elaidic acid; OA, oleic acid; $\mathrm{MbCD}$, methyl- $\beta$-cyclodextrin; EGF, epidermal growth factor; GPR, G-protein coupled receptor; EGFR, EGF receptor; Src, protooncogene tyrosine-protein kinase Src; APC, adenomatous polyposis coli; NS, nucleostemine; ERK, extracellular signal-regulated kinase; NICD, Notch intracellular domain; FDA, Food and Drug Administration; EMT, epithelial-mesenchymal transition

Key words: signal transactivation, wnt, cancer stem cell, fatty acid

\section{Introduction}

Colorectal cancer (CRC) is the third leading cause of cancer death in Japan (1). The number of patients with CRC and the number of deaths due to CRC have increased in Japan because of the popularity of the western lifestyle $(2,3)$. Some dietary ingredients, such as fatty acids, are risk factors for CRC (4). Linoleic acid (LA) increases the risk of colorectal carcinogenesis by generating prostaglandin E2, which induces chronic persistent inflammation of the mucosa (5). LA also induces dormancy in CRC cells (6). Trans fatty acids (TFAs) have been evaluated as a possible CRC risk for decades (7); however, recent studies have clearly demonstrated that TFAs are associated with colon carcinogenesis. Patients who do not use non-steroidal anti-inflammatory drugs and postmenopausal women showed higher CRC risks from TFAs (8). Compared with patients in the lowest quartile of TFA consumption, those in the highest quartile showed an increased odds ratio of 1.86 for the prevalence of colorectal adenomas (9). Moreover, elaidic acid (EA), the major TFA (10), is increased in the plasma of colon adenoma patients (11). Additionally, EA promotes colon carcinogenesis in Min mice (12) and induces the proliferation of Ehrlich ascites sarcoma cells (13). Thus, TFAs, particularly EA, possess carcinogenic properties; however, the related intracellular signals have not been fully determined.

Long-chain fatty acids (LCFAs) bind to specific membranebound receptors, namely G-protein coupled receptor 40 (GPR40) and GPR120 (14). GPR40 and GPR120 bind to various LCFAs. EA is an LCFA containing 18 carbon chain with a trans configuration and is a structural isomer of oleic acid (OA). OA also binds to GPR40 and 120, which is coupled with $\mathrm{Gq}$, to stimulate calcium ion influx and inositol phosphate synthesis in smooth muscle cells (15), inducing smooth muscle contraction. OA inhibits c-Jun N-terminus kinase and nuclear factor $\kappa \mathrm{B}$ signaling (16), which suppress tumor necrosis factor $\alpha$-induced insulin resistance and inflammation.

However, no information is available regarding EA receptors. EA is a well-known dietary risk factor of cardiovascular disorders such as atherosclerosis and myocardial infarction $(17,18)$. OA is present at low concentrations in CRC tissues (19), whereas EA is increased in the plasma of colon adenoma patients (11). OA exerts protective effects against 
cardiovascular disorders by decreasing low-density lipoprotein cholesterol levels (20). In contrast, EA exerts proinflammatory effects $(16,21)$, suggesting the presence of different intracellular signaling pathways for EA and OA.

In the present study, we examined the effect of EA on the metastasis of CRC cells and determined the receptors and signaling pathways of EA to explain its role in CRC.

\section{Materials and methods}

Cell culture and reagents. Mouse colon cancer cell line CT26 was kindly provided by Dr I. J. Fidler (MD Anderson Cancer Center, Houston, TX, USA) and human colon cancer cell line HT29 was purchased from Dainihon Pharmacy, Co., Ltd., (Tokyo, Japan). Cells were routinely maintained in Dulbecco's modified Eagle's medium (DMEM; Sigma-Aldrich, St. Louis, MO, USA) supplemented with $10 \%$ fetal bovine serum (FBS; Sigma-Aldrich) in $5 \% \mathrm{CO}_{2}$ at $37^{\circ} \mathrm{C}$. Cell morphology was evaluated daily by performing microscopic examination. Each cell line was routinely tested for mycoplasma contamination by performing genomic PCR. Before manuscript submission, viability of each cell line was tested by performing trypan blue exclusion assay.

EA (CAS no. 112-79-8; Wako Pure Chemical Industries Ltd., Osaka, Japan), OA (CAS no. 112-80-1; Wako Pure Chemical Industries), methyl- $\beta$-cyclodextrin (MbCD; SigmaAldrich), human epidermal growth factor (EGF; Peprotech EC Ltd., London, UK), PP1 (SRC inhibitor; Sigma-Aldrich), Wnt inhibitor (IWP3; Miltenyi Biotec GmbH, Bergisch Gladbach, Germany) (22), ERK inhibitor (3-[2-aminoethyl]-5-[\{4ethoxyphenyl\}methylene]-2,4-thiazolidinedione; Santa Cruz Biotechnology, Santa Cruz, CA, USA) and p38 inhibitor (SB 203580; Abcam, Cambridge, MA, USA) were purchased.

Assessment of cell growth and apoptosis. The cells (1 $10^{4} /$ well) were seeded in a 12-well dish. Cell growth was assessed by performing 3-(4,5-dimethylthiazol-2-yl)-2,5-diphenyltetrazolium bromide (MTT) assay or by counting the number of cells with autocytometer (Sysmex, Kobe, Japan) after $48 \mathrm{~h}$, as previously described (23). Apoptosis and necrosis were assessed using an apoptosis/necrosis detection kit (Enzo Life Sciences, Plymouth Meeting, PA, USA). Annexin V-EnzoGold and 7-AAD present in the kit fluorescently label apoptotic and necrotic cells, respectively. Apoptosis or necrosis was determined by examining 2000 cells under a fluorescent microscope (Leica Microsystems, Tokyo, Japan). All the experiments were performed in triplicate.

Sphere assay. The cells (1 $1 \times 10^{7} /$ well) were seeded on stem cell medium II (Sigma-Aldrich) in a bacteriological $3.5-\mathrm{cm}$ dish (Becton-Dickinson Labware, Bedford, MA, USA) for 1 week. Number of spheres formed was counted by performing darkfield microscopy (Nikon, Tokyo, Japan).

Reverse transcriptase-polymerase chain reaction. Mouse mRNA expression was assessed by performing reverse transcriptase-polymerase chain reaction (RT-PCR) with $0.5 \mu \mathrm{g}$ total RNA extracted using RNeasy kit (Qiagen, Hilden, Germany). Primer sets used for amplifying mouse $C D 133$ and nucleostemin (NS; also known as guanine nucleotide-binding protein-like 3) are as follows: CD133 (NCBI reference sequence: NM_008935) forward, 5'-GAA AAG TTG CTC TGC GAA CC-3' and CD133 reverse 5'-TCT CAA GCT GAA AAG CAG CA-3'; NS (NCBI reference sequence: NM_153547) forward, 5'-CAG GAT GCT GAC GAT CAA GA-3' and $N S$ reverse 5'-TTG ATT GCT CAG GTG ACA GC-3'. These primer sets were synthesized by Sigma Genosys (Ishikari, Japan). PCR products obtained were electrophoresed on a $2 \%$ agarose gel and stained with ethidium bromide. $\beta$-actin $(A C T B)$ mRNA (GenBank accession no. NM_001101) was amplified for use as an internal control.

Immunohistochemical analysis. Cell spheres formed were fixed in $10 \%$ buffered formalin for $12 \mathrm{~h}$ at $4^{\circ} \mathrm{C}$ and were embedded in $1 \%$ agar. The agar specimens were processed into a paraffin-embedded block and $4-\mu \mathrm{m}$-thick sections were cut using a microtome. Consecutive tissue sections were immunohistochemically stained by following the immunoperoxidase technique, as previously described (24). Immunoblotting analysis was performed using $0.2 \mu \mathrm{g} / \mathrm{ml}$ anti-MIB1 and anti-NS antibodies (Dako Corp., Carpinteria, CA, USA) after performing antibody retrieval, in which the sections were heated three times in phosphate buffer $(\mathrm{pH}$ 6.0) in a microwave for $15 \mathrm{~min}$ each. Immunoreactivity was determined using $0.2 \mu \mathrm{g} / \mathrm{ml}$ secondary antibodies (Medical and Biological Laboratories, Co., Ltd., Nagoya, Japan). Color development was performed using diamine benzidine hydrochloride (Dako) and counterstaining was performed using Mayer's hematoxylin (Sigma-Aldrich). The number of NS- or MIB1-positive nuclei were counted and expressed as the mean number of positive nuclei from 50 high-power fields of tumor specimens.

Animal models. BALB/c male mice (4-weeks old) were purchased from Japan SLC (Shizuoka, Japan). The mice were maintained according to the institutional guidelines approved by the Committee for Animal Experimentation of Nara Medical University and the current regulations and standards established by the Ministry of Health, Labor and Welfare. Each experimental group included five mice.

A subcutaneous tumor model was established by inoculating CT26 cells ( $1 \times 10^{7}$ cells) suspended in Hank's balanced salt solution (Sigma-Aldrich) into the scapular subcutaneous tissue of the mice, which were fed with standard diet (CE-2; CLEA Japan, Tokyo, Japan). The fatty acid composition did not contain TFAs). For analyzing cancer metastasis to the liver, lungs and peritoneum, cancer cells $\left(1 \times 10^{6}\right.$ cells $)$ were inoculated into the spleen, tail vain and peritoneal cavity, respectively. EA or OA (10 $\mathrm{mg} /$ mouse) was administered by gavage every 7 days, and metastatic status was assessed after 4 weeks. The dosage of $10 \mathrm{mg} / \mathrm{mouse} /$ week (equivalent to $0.5 \mathrm{~g} / \mathrm{kg}$ body weight $/$ week) is approximately equal to $0.53 \mathrm{~g} / \mathrm{kg}$ body weight/week, which is according to the Nutrition Facts label of foods $4.6 \mathrm{~g}$ per day (25). Peritoneal metastasis was determined by measuring the weight of the mesenterium with tumors, which was separated from the intestinal tube. The liver and lung metastases were determined by counting the number of tumors on organ surface.

Western blot analysis. Whole-cell lysates were prepared as previously described (24). Cell fractions were extracted 
by processing the cells with Cell Fractionation kit (Abcam), according to the manufacturer's instruction.

Proteins $(50 \mu \mathrm{g})$ present in cell lysates were separated by performing sodium dodecyl sulfate-polyacrylamide gel electrophoresis on $12.5 \%$ gels and were electrotransferred onto nitrocellulose membranes for immunoblotting analysis. The membranes were incubated with primary antibodies, followed by incubation with peroxidase-conjugated $\operatorname{IgG}$ antibodies (MBL). Tubulin antibody (Proteintech Group, Inc., Rosemont, IL, USA) was used to measure the amount of protein loaded per lane. Immune complexes were visualized using CSA system (Dako). Antibodies against GPR40 and GPR120 (Abnova Corp., Taipei City, Taiwan); EGFR, E-cadherin (ECD) and $\beta$-catenin (Transduction Laboratories, Lexington, KY, USA); phosphorylated EGFR, NS, phosphorylated ERK1/2 $\left(\mathrm{pThr}^{204}\right)$, phosphorylated SRC (pTyr $\left.{ }^{530}\right)$, Notch1, APC, Wnt5a and CD44 (Santa Cruz Biotechnology); phosphorylated p38 (pThr ${ }^{180} /$ pTyr $^{182}$; Cusabio, College Park, MD, USA); CD133 and Snail1 (pSer246) (Biorbyt, Cambridge, UK); and phosphorylated $\beta$-catenin (pSer ${ }^{33 / 37}$; LifeSpan Biosciences, Inc., Seattle WA, USA) were used as primary antibodies.

Small interfering RNA. Stealth Select RNAi oligonucleotides (siRNAs) against human and mouse GPR40 and GPR120 and against mouse EGFR were purchased from Santa Cruz Biotechnology. AllStars Negative Control siRNA was used as a control (Qiagen, Valencia, CA, USA). Cells were transfected with $20 \mathrm{nM}$ siRNA by using Lipofectamine 2000 (Invitrogen Corp., Carlsbad, CA, USA), according to the manufacturer's recommendations.

Surface expression assay. Surface expression assay was performed to determine the effect of agonist-mediated GPR internalization. Briefly, monolayer-cultured CT26 cells in a 96-well dish were preincubated with peroxidase-labeled antibody against GPR40 or GPR120, followed by treatment with EA, OA $(35 \mu \mathrm{M})$ or vehicle. At each time-point, the cells were counted by performing the MTT assay and were fixed in $4 \%$ paraformaldehyde for $15 \mathrm{~min}$ at room temperature. Surface levels of GPRs were measured by performing tetramethylbenzene reaction, followed by treatment with $\mathrm{H}_{2} \mathrm{SO}_{4}$ and measurement of absorbance at $450 \mathrm{~nm}$.

Statistical analysis. Statistical analyses of experimental data were performed by unpaired Student's t-test and one-way ANOVA with post hoc test. Normal distribution of the data samples was confirmed by the Kolmogorov-Smirnov normality test. A two-sided $\mathrm{P}<0.05$ was considered statistically significant.

\section{Results}

EA enhances metastasis. As shown in Fig. 1, CT26 mouse CRC cells were inoculated into the subcutaneous tissue (Fig. 1A), spleen (liver metastasis) (Fig. 1B), tail vein (lung metastasis) (Fig. 1C) and peritoneum (Fig. 1D) of BALB/c mice by gavage administration with $\mathrm{EA}, \mathrm{OA}$, or control vehicle. EA-treated mice showed higher growth of subcutaneous tumors and metastases to the liver, lungs and peritoneum than control mice. In contrast, OA-treated mice only showed increased

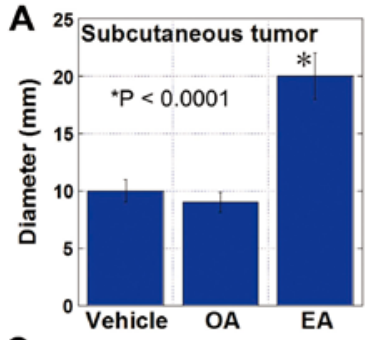

C
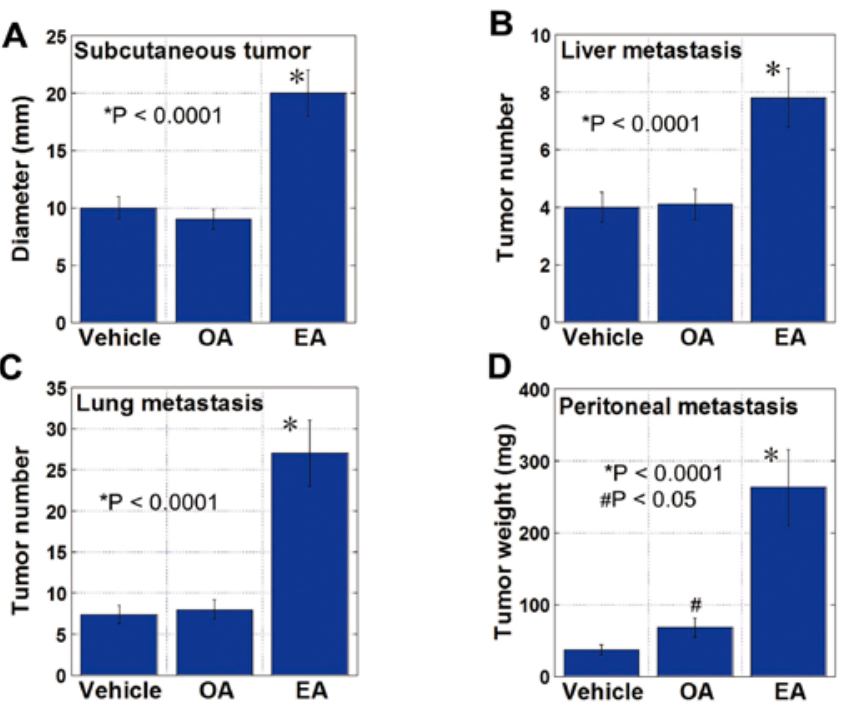

D
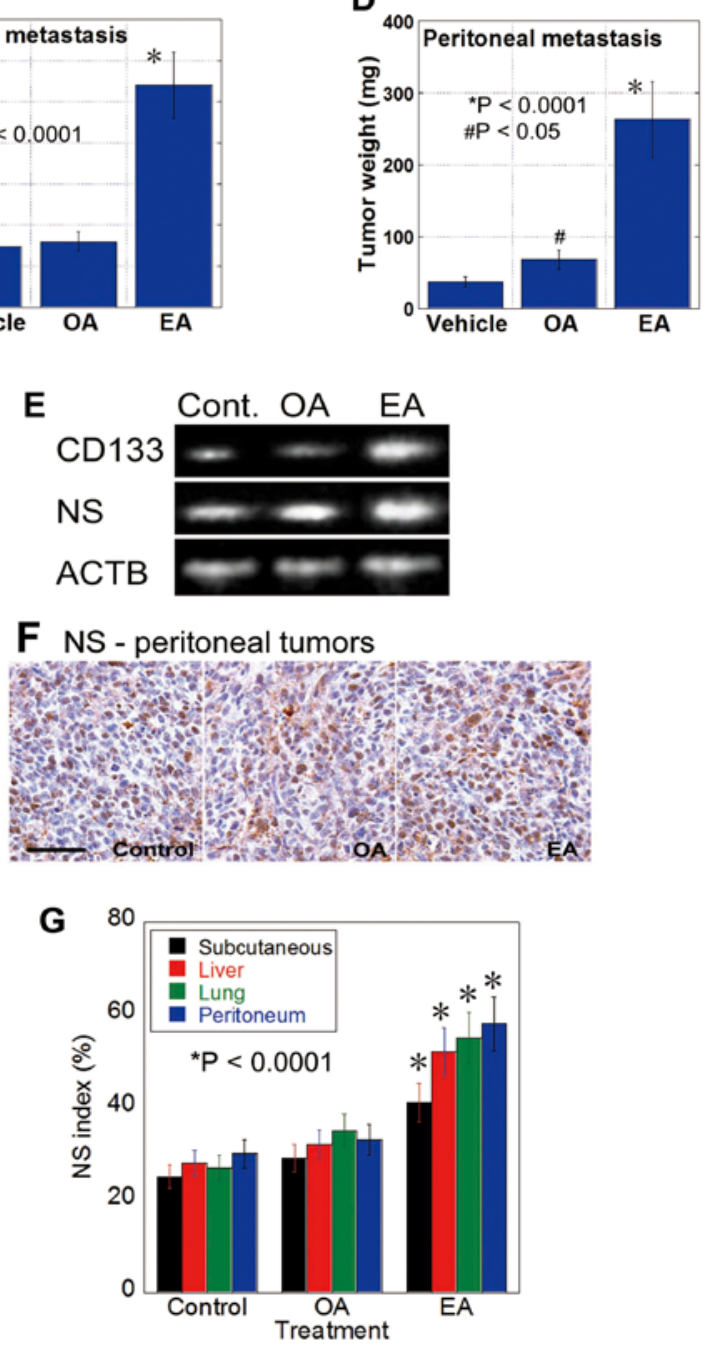

Figure 1. Gavage administration of EA enhances the metastasis of CT26 cells. (A-D) Metastasis of CT26 mouse CRC cells in mice treated with EA or OA by gavage $(10 \mathrm{mg} / \mathrm{mouse}$ in $30 \%$ ethanol) or vehicle (30\% ethanol). Tumors were assessed at 4 weeks after cancer cell inoculation. Tumor diameter (A), tumor number at the surface of the liver (B) and lung (C), and tumor weight (D) of the mesenterium were measured for subcutaneous tumor, metastases to the liver, lung and peritoneum, respectively. Error bar, SD. *Statistical difference from control was calculated by the unpaired Student's t-test. (E) Expression of CD133 and NS in subcutaneous tumors at week 4 was examined by RT-PCR. (F) Expression of NS was also examined by immunostaining peritoneal tumors at week 4; scale bar, $100 \mu \mathrm{m}$. (G) NS index, i.e., the percentage of NS-positive nuclei in tumor cells, was calculated by examining 500 tumor cells by immunostaining. Error bar, SD. *Statistical difference from control was calculated by the unpaired Student's t-test.

peritoneal metastasis compared with the control mice. Tumors of EA-treated mice showed increased mRNA expression of CD133 and NS (Fig. 1E). Moreover, the number of NS-positive tumor cells increased in the tumors of EA-treated mice but not 

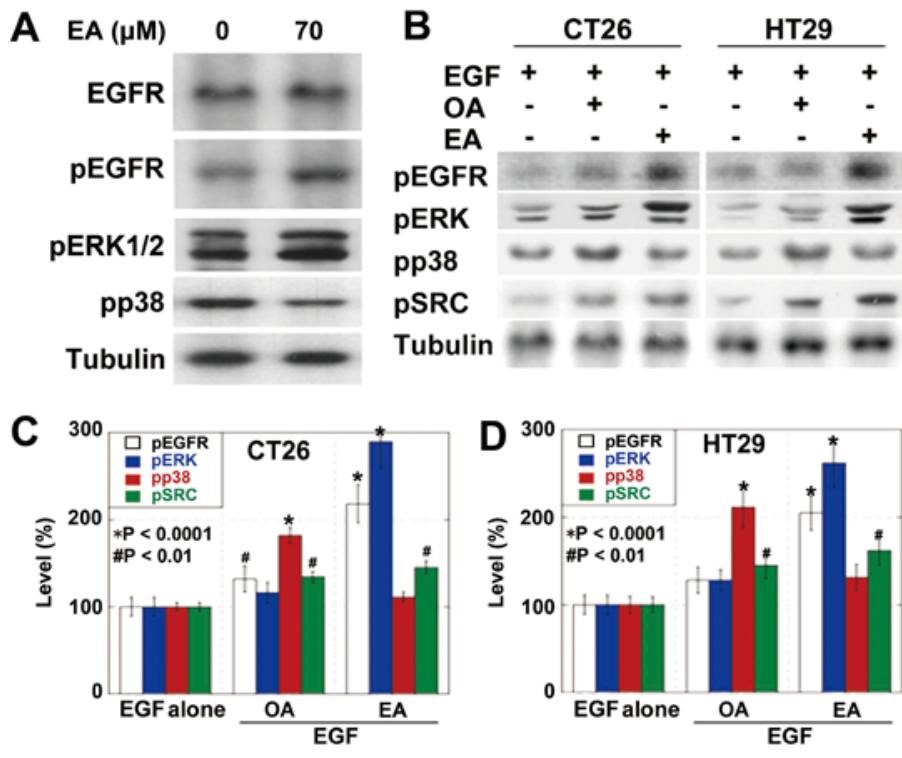

Figure 2. Intracellular signaling of EA. (A) EGFR, phosphorylated EGFR, ERK1/2 and p38 phosphorylation in EA-treated CT26 cells. (B-D) Effect of EA or OA $(70 \mu \mathrm{M})$ on EGF-induced EGFR, ERK1/2, p38 and SRC phosphorylation in CT26 and HT29 cells was examined. EGF (10 ng/ml) was added to increase the basal phosphorylation levels of EGFR. (B) Western blot analysis. Tubulin served for an internal control. (C and D) Semi-quantification of the results of western blot analysis by performing densitometric scanning. Each bar represents mean \pm SD of three independent experiments. "Statistical difference from EGF alone was calculated by the unpaired Student's t-test.

A

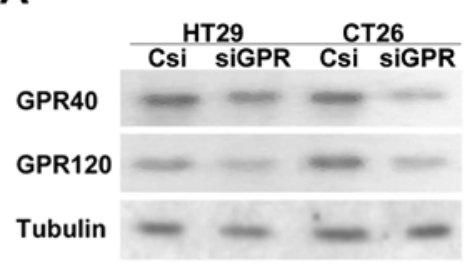

C

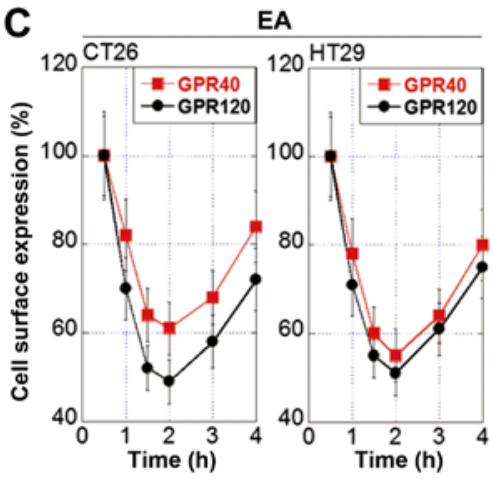

E

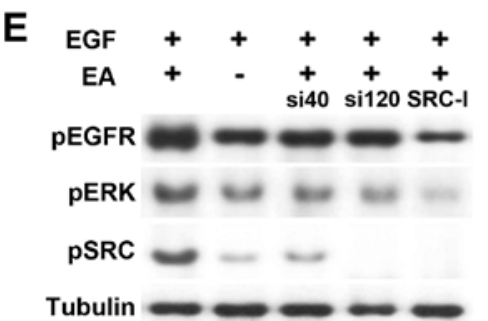

B

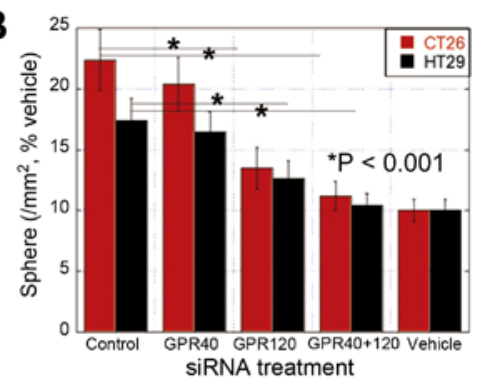

D

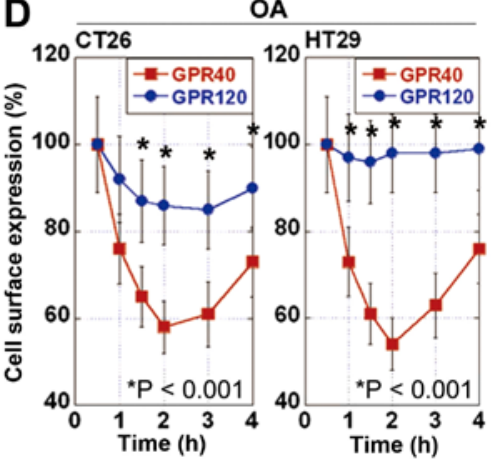

$\mathbf{F}$

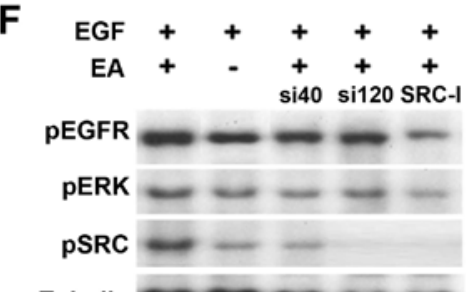

Tubulin

Figure 3. Receptors of EA. (A) Protein expression of long chain fatty acid receptors GPR40 and GPR120 in CRC cells transfected with control siRNA (Csi) or siRNA against the gene encoding GPR40 or GPR120. (B) Effect of the knockdown of genes encoding GPR40 and/or GPR120 on the number of sphereforming cells. Error bar, SD of three independent experiments. "Statistical difference between GPR40 and GPR120 was calculated by the unpaired Student's t-test. (C and D) Surface expression assay for assessing the internalization of GPR40 and GPR120 in CRC cells treated with EA or OA (70 $\mu$ M). Error bar, SD. "Statistical difference from control was calculated by the unpaired Student's t-test. (E and F) Effect of SRC inhibitor (PP1, $0.05 \mu \mathrm{M})$ and knockdown of the gene encoding GPR40 (si40) or GPR120 (si120) on EA-induced EGFR phosphorylation (70 $\mu \mathrm{M})$ in CRC cells. EGF (10 ng/ml) was added for increasing the basal phosphorylation levels of EGFR. Tubulin was used as an internal control. 

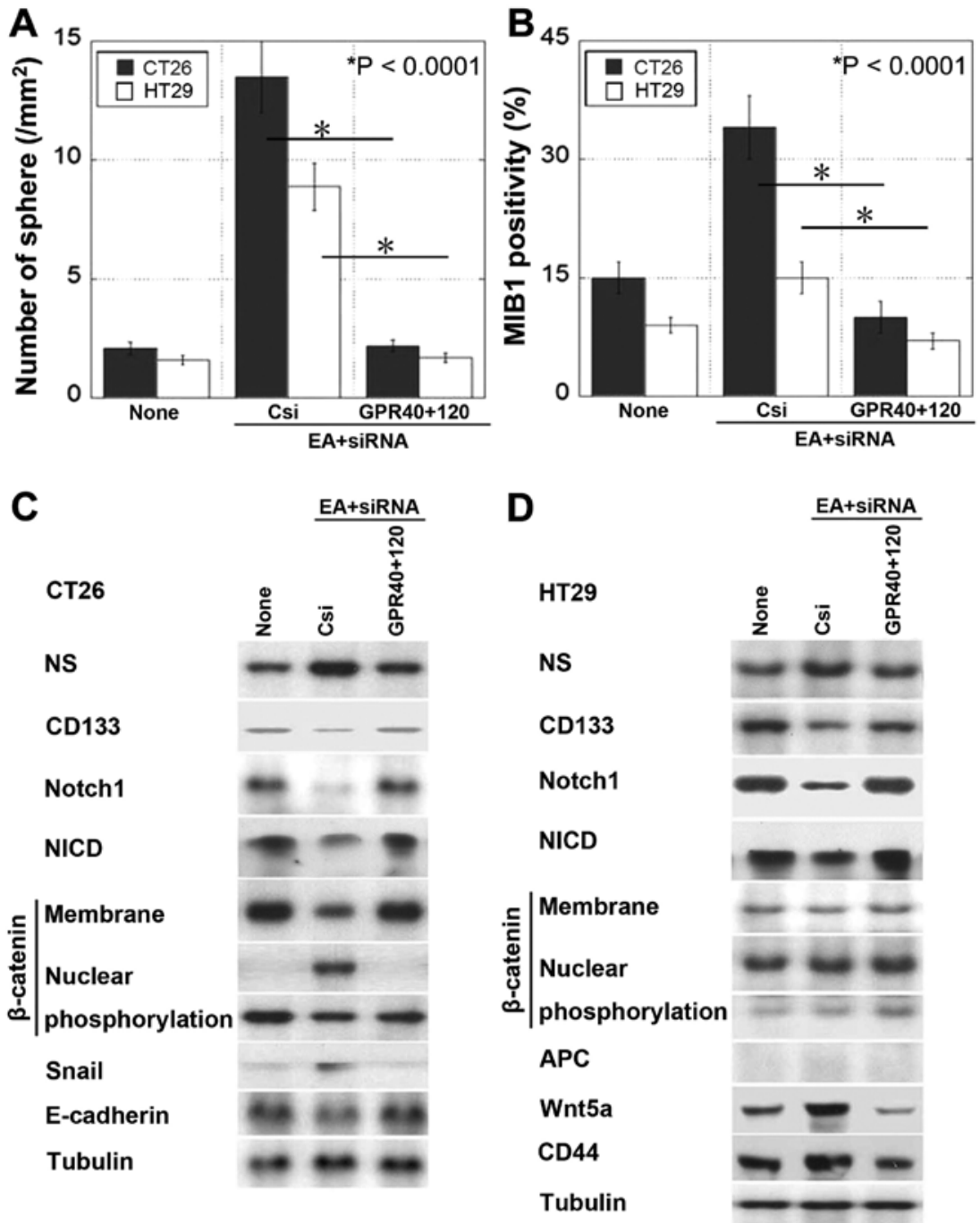

Figure 4. Alteration of stemness-associated factors by EA. (A and B) Effect of EA and/or knockdown of genes encoding GPRs on the number of sphere and percentage of MIB1-positive cells to 500 sphere-forming cells. Csi, control siRNA. Error bar, SD. "Statistical was calculated by the unpaired Student's t-test. (C) Effect of EA on Notch and canonical Wnt signaling and EMT in CT26 cells. (D) Effect of EA on Notch and canonical and non-canonical Wnt signaling in HT29 cells. NS, nucleostemine; NICD, Notch intracellular domain; memb, $\beta$-catenin in membrane fraction; nuclear, $\beta$-catenin in nuclear fraction; phopho, Ser33/37 phosphorylated $\beta$-catenin; APC, adenomatous polyposis coli. Tubulin was used as an internal control.

in the tumors of control or OA-treated mice (Fig. $1 \mathrm{~F}$ and $\mathrm{G}$ ). These results suggest that EA increases the stemness of CT26 cells to enhance their metastatic potential.

Intracellular signaling pathways of EA. Next, we examined the intracellular signals of EA (Fig. 2). EA treatment induced the phosphorylation of EGFR, ERK1/2 but not p38 (Fig. 2A). The effects of EA and OA on EGFR activation were compared (Fig. 2B-D). Phosphorylation of EGFR and ERK1/2 induced by OA was less pronounced than that induced by EA in both CRC cells. In contrast, phosphorylation of $\mathrm{p} 38$ induced by OA was more pronounced than that induced by EA. Importantly, EA treatment induced SRC phosphorylation at higher levels than those by OA treatment in both cell types.

Receptor of EA. GPR40 and GPR120 function as receptors of long chain fatty acids (26). We observed that CRC cells expressed different levels of GPR40 and GPR120 (Fig. 3A) and that CT26 cells showed higher GPR120:GPR40 ratio (5.3) than HT29 cells (1.2).
We knocked down genes encoding GPR40 and/or GPR120 and examined the effects of gene knockdown on the number of sphere-forming cells (Fig. 3B). Knockdown of the gene encoding GPR120 decreased the number of sphere-forming cells more profoundly than the knockdown of the gene encoding GPR40. Concurrent knockdown of both GPR40- and GPR120-encoding genes blocked EA-induced increase in the number of sphere-forming cells.

Binding affinity of EA to GPR40 or GPR120 was confirmed by examining the cell surface expression of these GPRs. Cell surface expression of both GPR40 and GPR120 was downregulated by internalization for $2 \mathrm{~h}$ after EA treatment, after which it recovered (Fig. 3C). Results of the surface expression assay indicate that unlike EA, OA may preferentially bind to GPR40 (Fig. 3D).

EGFR transactivation by EA. Proto-oncogene tyrosine protein kinase (SRC) plays an important role in OA-induced EGFR transactivation (27). Therefore, we examined the role of SRC in EA-induced signal transduction. We observed that 

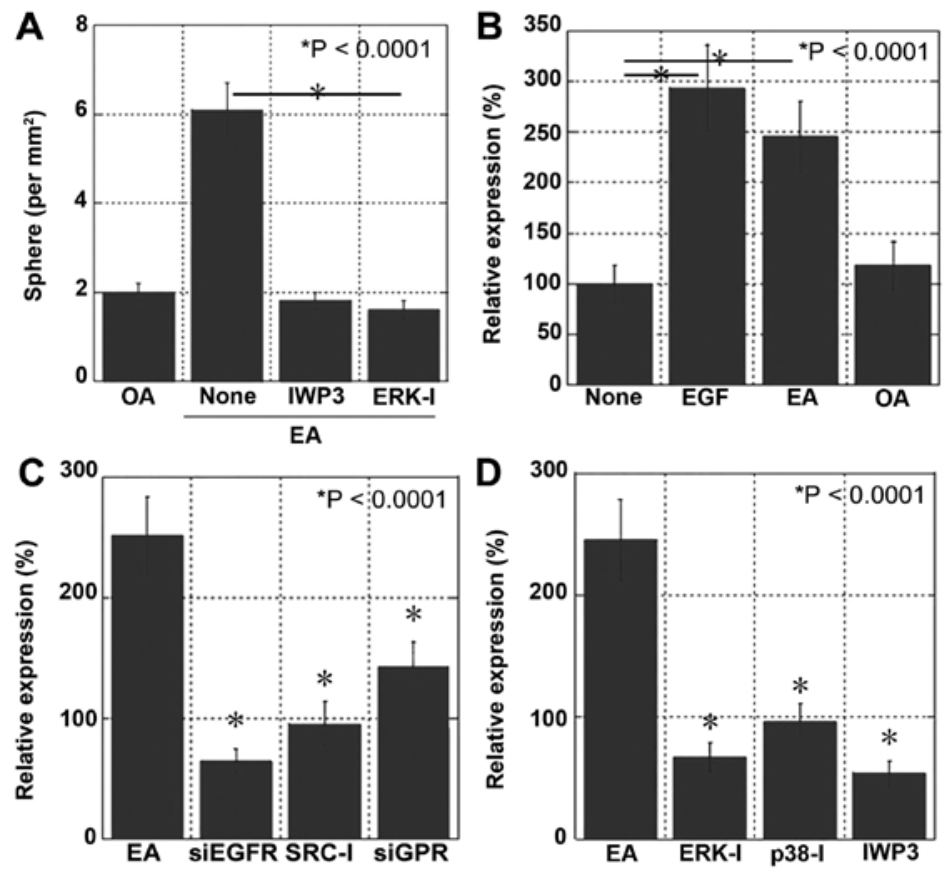

Figure 5. EA signaling and stemness. (A) Effect of inhibitors of Wnt signaling (IWP3, $2 \mu \mathrm{M})$ and ERK1/2 $(10 \mu \mathrm{M})$ on sphere formation by CT26 cells (B-D) Alteration in NS expression was examined in CT26 cells. (B) NS levels by treatment with EGF, EA and OA. (C) NS levels with inhibition of EGFR transactivation by knockdown (siEGFR) of EGFR; by SRC inhibitor (PP1, $0.05 \mu \mathrm{M}$ ); by knockdown (siGPR) of GPR40 and GPR120. (D) NS levels with inhibition of EGFR signaling by using inhibitors of ERK $(10 \mu \mathrm{M})$, p38 (SB203580, $10 \mu \mathrm{M})$ or Wnt (IWP3, $2 \mu \mathrm{M})(\mathrm{D})$. Each bar; mean \pm SD of three independent experiments. "Statistical difference between two groups (A and B) or from EA (C and D) was calculated by the unpaired Student's t-test.

SRC inhibition suppressed the phosphorylation of EGFR and ERK1/2 in CT26 cells after concurrent treatment with EA and EGF (Fig. 3E). Moreover, knockdown of the gene encoding GPR40 or GPR120 decreased the phosphorylation of EGFR and ERK1/2 (Fig. 3E). Knockdown of the gene encoding GPR120 inhibited the phosphorylation of EGFR and ERK1/2 more profoundly than the knockdown of the gene encoding GPR40. We also observed these effects in HT29 cells (Fig. 3F). These findings suggest that EA activates GPR120 and (to a lesser extent) GPR40 in CRC cells for transactivating EGFR through SRC.

Effect of EA on cancer stem cell-associated signals. Next, we examined sphere formation as a feature of cancer stem cells (Fig. 4A and B). We observed that the number of spheres and MIB1-positive proliferating cells increased after EA treatment and decreased after knocking down the genes encoding GPR40 and GPR120. Treatment of CT26 cells with EA altered stem cell marker expression, i.e., it increased NS expression and decreased CD133 expression (Fig. 4C). Moreover, EA treatment decreased Notch and Notch intracellular domain (NICD) expression and induced $\beta$-catenin nuclear translocation associated with EMT, as indicated by the upregulation of Snail expression and downregulation of E-cadherin expression. Thus, EA signaling inhibited Notch signaling and activated canonical Wnt pathway in CT26 cells. In contrast, EA treatment of HT29 cells harboring truncated APC (28), which showed constitutively activated canonical Wnt signaling, increased NS expression and decreased CD133 expression (Fig. 4D). In addition, EA treatment of HT29 cells decreased Notch and NICD expression and increased Wnt5a and CD44 expression. These results indicate that EA signaling inhibits
Notch signaling and activates non-canonical Wnt signaling in HT29 cells.

EA signaling and stemness. To confirm that EA induced the stemness and metastatic ability of CRC cells, we examined the effects of Wnt and ERK1/2 inhibitors by performing the sphere assay (Fig. 5A). Treatment with Wnt and ERK1/2 inhibitors completely abolished EA-induced increase in sphere number and liver metastasis.

To confirm EA signaling pathway, NS expression in CT26 cells was examined at the ligand (Fig. 5B), receptor (Fig. 5C), and post-receptor signaling levels (Fig. 5D). At the ligand level, EA treatment increased NS expression to a similar level as that after EGF treatment, suggesting that EGFR transactivation induced by EA was as effective as that induced by EGF. At the receptor level, inhibition of the crosstalk linker SRC and knockdown of GPR40 and GPR120 suppressed EA-induced NS expression, which was as effective as that induced by the knockdown of EGFR. At the post-receptor signaling level, inhibition of ERK1/2, p38 and Wnt suppressed EA-induced NS expression.

\section{Discussion}

The results of the present study indicate that EA strongly enhances cancer metastasis by increasing the stemness and the epithelial-mesenchymal transition (EMT) of colon cancer cells.

GPRs function as receptors of LCFAs. Particularly, GPR40 and GPR120 are receptors for unsaturated LCFAs such as OA or LA (26). In the present study, the binding of EA to GPR40 and GPR120 was confirmed in surface protein internalization 
assays. Knockdown of the genes encoding these two receptors did not completely suppress the EA-induced increase in the proliferation of cancer spheres. However, further studies are needed to examine other potential receptors that effectively prevent EA-induced proliferation of cancer spheres.

GPR40 and GPR120 perform different functions under different conditions. In islet $\beta$ cells, GPR40 activation increases insulin resistance, whereas GPR120 activation increases insulin sensitivity (29). The motility and invasion of melanoma, lung and pancreatic cancer cell lines are enhanced by GPR40, but suppressed by GPR120 (30-32).

The results of the present study indicate that GPR40 and GPR120 are cooperatively involved in EA-induced EGFR transactivation. In OA-treated MCF7 cells, GPR40 and GPR120 induce EGFR transactivation (27). Transactivation may involve the participation of both GPR40 and GPR120. OA-activated EGFR induces activator protein 1 to enhance cell proliferation and matrix metalloproteinase expression (27). We observed that EA crosstalk from GPRs to EGFR was mediated by SRC. A previous study reported that the crosstalk between GPRs and EGFR activated phospholipases C, D and A2, which subsequently activated protein kinase $C$, extracellular receptor kinase and Akt (33). Moreover, our data showed that EA-activated EGFR induced Wnt to enhance stemness and EMT. The causes of the underlying differences downstream of transactivated EGFR by EA and OA are unclear. Therefore, further studies are necessary to explore other receptors or signaling pathways involved downstream of EGFR.

In wild-type APC-containing CT26 cells, EA signaling through EGFR induced EMT and increased stemness by activating the canonical Wnt pathway. In contrast, in APC-null HT29 cells, EA-EGFR signaling increased stemness by activating the non-canonical Wnt pathway. This is consistent with our previous findings that EGFR increased stemness by activating Wnt signaling through the nuclear translocation of $\beta$-catenin (22). In the present study, we found that EA, GPRs, SRC-EGFR signaling, and phosphorylation of extracellular receptor kinase 1/2 and p38 were involved in EA-induced Wnt activation. Thus, our results indicate that EMT and stemness enhance the metastatic potential of CRC cells. Notably, EA treatment upregulated NS, but not CD133 and increased MIB1positive stem cells in the sphere. These findings suggest that cancer stem cells activated by EA are heterogeneous and show a preference for proliferative stem cells. Moreover, our results suggest that inhibition of EA signaling is a relevant therapeutic strategy for inhibiting EA-induced metastasis of CRC cells.

EA have been reported as EA incorporated into mitochondrial membrane reducing early closing and refractoriness of voltage-dependent anion channels (34). EA affects global DNA methylation by inducing a pro-inflammatory transcriptional profile different from that induced by OA in vitro. Moreover, maternal EA administration induces DNA hypermethylation in mouse progeny (35). The macrophages from rats fed a diet containing hydrogenated fat showed upregulation of cytosolic phospholipase A2, nuclear factor- $\kappa \mathrm{B}$ p65 and Toll-like receptor- 2 and -4 , and downregulation of peroxisome proliferator activated receptor- $\gamma$ and adiponectin receptor-1 and -2 , enhancing pro-inflammatory phenotypes (36). In human, administration of an EA-rich diet to healthy volunteers resulted in decreased mitogen-induced
CD69 expression on $\mathrm{CD} 8^{+} \mathrm{T}$ cells and phagocytic activity on neutrophils (37). Such epigenetic, pro-inflammatory and anti-cytotoxic immune properties may affect carcinogenic and pro-metastatic potential of EA in addition to enhancing the stemness of cancer cells.

Gavage administration of EA had a marked pro-metastatic effect in the syngeneic tumor model using CT26 CRC cells and $\mathrm{BALB} / \mathrm{c}$ mice. Because the mice were fed with standard diet containing no TFAs, the enhanced metastatic ability is thought to be associated with EA administration. EA is a major component of dietary TFAs (10), and the dosage used in the experiments was in accordance with the recommendations by the Nutritional Label in America in 2003 (25). Indeed, our data showed that EA strongly promotes cancer metastasis. Metastasis is a major problem in cancer treatment and makes it difficult to cure cancer (38). Prevention of metastasis should be emphasized in the prevention of carcinogenesis. The ubiquitous usage of TFAs in western-style diets suggests that TFAs play a major role in the carcinogenesis and metastasis of CRC. The control of TFA-associated cancer development and progression is urgently needed.

\section{Acknowledgements}

The authors thank Ms. Tomomi Masutani for her expert assistance with the preparation of this manuscript. The present study was supported by the MEXT KAKENHI grant nos. 13200228, 14478268, 13394212, 13209774 and 16675788.

\section{References}

1. Wakao F, Nishimoto H, Kataonoda K, Tsukuma $\mathrm{H}$ and Mikami $\mathrm{H}$ (eds). Cancer Statistics in Japan, 2013. National Cancer Research Institute, Tokyo, 2013.

2. Kimura Y, Kono S, Toyomura K, Nagano J, Mizoue T, Moore MA, Mibu R, Tanaka M, Kakeji Y, Maehara Y, et al: Meat, fish and fat intake in relation to subsite-specific risk of colorectal cancer: The Fukuoka Colorectal Cancer Study. Cancer Sci 98: 590-597, 2007.

3. Mizoue T, Tanaka K, Tsuji I, Wakai K, Nagata C, Otani T, Inoue $\mathrm{M}$ and Tsugane $\mathrm{S}$; Research Group for the Development and Evaluation of Cancer Prevention Strategies in Japan: Alcohol drinking and colorectal cancer risk: An evaluation based on a systematic review of epidemiologic evidence among the Japanese population. Jpn J Clin Oncol 36: 582-597, 2006.

4. Bultman SJ: Interplay between diet, gut microbiota, epigenetic events, and colorectal cancer. Mol Nutr Food Res: May 3, 2016. (Epub ahead of print) doi: 10.1002/mnfr.201500902.

5. Moonen HJ, Dommels YE, van Zwam M, van Herwijnen MH, Kleinjans JC, Alink GM and de Kok TM: Effects of polyunsaturated fatty acids on prostaglandin synthesis and cyclooxygenase-mediated DNA adduct formation by heterocyclic aromatic amines in human adenocarcinoma colon cells. Mol Carcinog 40: 180-188, 2004.

6. Ohmori H, Sasahira T, Fujii K, Luo Y, Shimomoto T and Kuniyasu H: Linoleic acid-induced growth suppression induces quiescent cancer cell nests in nude mice Pathobiol 75: 226-232, 2008.

7. Valenzuela A and Morgado N: Trans fatty acid isomers in human health and in the food industry. Biol Res 32: 273-287, 1999.

8. Slattery ML, Benson J, Ma KN, Schaffer D and Potter JD: Transfatty acids and colon cancer. Nutr Cancer 39: 170-175, 2001.

9. Vinikoor LC, Schroeder JC, Millikan RC, Satia JA, Martin CF, Ibrahim J, Galanko JA and Sandler RS: Consumption of transfatty acid and its association with colorectal adenomas. Am J Epidemiol 168: 289-297, 2008.

10. Precht D and Molkentin J: Trans fatty acids: Implications for health, analytical methods, incidence in edible fats and intake (a review). Nahrung 39: 343-374, 1995. 
11. Pickens CA, Lane-Elliot A, Comstock SS and Fenton JI: Altered saturated and monounsaturated plasma phospholipid fatty acid profiles in adult males with colon adenomas. Cancer Epidemiol Biomarkers Prev 25: 498-506, 2016.

12. Molin M, Berstad P, Benth JS, Alexander J, Paulsen JE and Almendingen K: Effect of different degrees of hydrogenated fish oil on intestinal carcinogenesis in Min ${ }^{+}$mice. Anticancer Res 33: 477-483, 2013.

13. Awad AB: Trans fatty acids in tumor development and the host survival. J Natl Cancer Inst 67: 189-192, 1981.

14. Hara T, Kashihara D, Ichimura A, Kimura I, Tsujimoto G and Hirasawa A: Role of free fatty acid receptors in the regulation of energy metabolism. Biochim Biophys Acta 1841: 1292-1300, 2014.

15. Mizuta K, Zhang Y, Mizuta F, Hoshijima H, Shiga T, Masaki E and Emala CW Sr: Novel identification of the free fatty acid receptor FFAR1 that promotes contraction in airway smooth muscle. Am J Physiol Lung Cell Mol Physiol 309: L970-L982, 2015.

16. Perdomo L, Beneit N, Otero YF,Escribano Ó,Díaz-Castroverde S, Gómez-Hernández A and Benito M: Protective role of oleic acid against cardiovascular insulin resistance and in the early and late cellular atherosclerotic process. Cardiovasc Diabetol 14: 75, 2015.

17. Remig V, Franklin B, Margolis S, Kostas G, Nece T and Street JC: Trans fats in America: A review of their use, consumption, health implications, and regulation. J Am Diet Assoc 110: 585-592, 2010.

18. Mozaffarian D, Aro A and Willett WC: Health effects of transfatty acids: Experimental and observational evidence. Eur J Clin Nutr 63 (Suppl 2): S5-S21, 2009.

19. Zhang J, Zhang L, Ye X, Chen L, Zhang L, Gao Y, Kang JX and Cai C: Characteristics of fatty acid distribution is associated with colorectal cancer prognosis. Prostaglandins Leukot Essent Fatty Acids 88: 355-360, 2013.

20. Huth PJ, Fulgoni VL III and Larson BT: A systematic review of high-oleic vegetable oil substitutions for other fats and oils on cardiovascular disease risk factors: Implications for novel higholeic soybean oils. Adv Nutr 6: 674-693, 2015.

21. Okada Y, Tsuzuki Y, Sato H, Narimatsu K, Hokari R, Kurihara C, Watanabe C, Tomita K, Komoto S, Kawaguchi A, et al: Trans fatty acids exacerbate dextran sodium sulphate-induced colitis by promoting the up-regulation of macrophage-derived proinflammatory cytokines involved in T helper 17 cell polarization. Clin Exp Immunol 174: 459-471, 2013.

22. Sasaki T, Kuniyasu H, Luo Y, Kato D, Shinya S, Fujii K, Ohmori H and Yamashita Y: Significance of epithelial growth factor in the epithelial-mesenchymal transition of human gallbladder cancer cells. Cancer Sci 103: 1165-1171, 2012.

23. Kuniyasu H, Yano S, Sasaki T, Sasahira T, Sone S and Ohmori H: Colon cancer cell-derived high mobility group 1/amphoterin induces growth inhibition and apoptosis in macrophages. Am J Pathol 166: 751-760, 2005.

24. Kuniyasu H, Oue N, Wakikawa A, Shigeishi H, Matsutani N, Kuraoka K, Ito R, Yokozaki H and Yasui W: Expression of receptors for advanced glycation end-products (RAGE) is closely associated with the invasive and metastatic activity of gastric cancer. J Pathol 196: 163-170, 2002.
25. U.S. Food and Drug Administration: FDA takes step to further reduce trans fats in processed foods, 2013.

26. Hirasawa A, Hara T, Katsuma S, Adachi T and Tsujimoto G: Free fatty acid receptors and drug discovery. Biol Pharm Bull 31: 1847-1851, 2008

27. Soto-Guzman A, Robledo T, Lopez-Perez M and Salazar EP: Oleic acid induces ERK1/2 activation and AP-1 DNA binding activity through a mechanism involving Src kinase and EGFR transactivation in breast cancer cells. Mol Cell Endocrinol 294: 81-91, 2008.

28. Yang J, Zhang W, Evans PM, Chen X, He X and Liu C: Adenomatous polyposis coli (APC) differentially regulates betacatenin phosphorylation and ubiquitination in colon cancer cells. J Biol Chem 281: 17751-17757, 2006.

29. Scheen AJ: Investigational insulin secretagogues for type 2 diabetes. Expert Opin Investig Drugs 25: 405-422, 2016.

30. Fukushima K, Takahashi K, Fukushima N, Honoki K and Tsujiuchi T: Different effects of GPR120 and GPR40 on cellular functions stimulated by 12-O-tetradecanoylphorbol-13-acetate in melanoma cells. Biochem Biophys Res Commun 475: 25-30, 2016.

31. Fukushima K, Yamasaki E, Ishii S, Tomimatsu A, Takahashi K, Hirane M, Fukushima N, Honoki K and Tsujiuchi T: Different roles of GPR120 and GPR40 in the acquisition of malignant properties in pancreatic cancer cells. Biochem Biophys Res Commun 465: 512-515, 2015.

32. Kita T, Kadochi Y, Takahashi K, Fukushima K, Yamasaki E, Uemoto T, Hirane M, Fukushima N, Honoki K and Tsujiuchi T: Diverse effects of G-protein-coupled free fatty acid receptors on the regulation of cellular functions in lung cancer cells. Exp Cell Res 342: 193-199, 2016.

33. Rozengurt E: Mitogenic signaling pathways induced by $\mathrm{G}$ protein-coupled receptors. J Cell Physiol 213: 589-602, 2007.

34. Tewari D and Bera AK: Modulation of the voltage-dependent anion channel of mitochondria by elaidic acid. Biochem Biophys Res Commun 477: 490-494, 2016.

35. Flores-Sierra J, Arredondo-Guerrero M, Cervantes-Paz B, Rodríguez-Ríos D, Alvarado-Caudillo Y, Nielsen FC, Wrobel K, Wrobel K, Zaina S and Lund G: The trans fatty acid elaidate affects the global DNA methylation profile of cultured cells and in vivo. Lipids Health Dis 15: 75, 2016.

36. Rao YP, Kumar PP and Lokesh BR: Molecular mechanisms for the modulation of selected inflammatory markers by dietary rice bran oil in rats fed partially hydrogenated vegetable fat. Lipids 51: 451-467, 2016.

37. Dlouhý P, Kucera P, Kraml P, Pompachová A, Potocková J, Smejkalová V, Mokrejs P, Jacek M and Andel M: Short-term dietary intake of C18:1 trans fatty acids decreases the function of cellular immunity in healthy young men. Ann Nutr Metab 53: 129-136, 2008.

38. Fidler IJ: The pathogenesis of cancer metastasis: The 'seed and soil' hypothesis revisited. Nat Rev Cancer 3: 453-458, 2003. 